with high hemoglobin levels according to the revised WHO classification. Leukemia. 2014;28(10):2092-2094.

45. Maslah N, Soret J, Dosquet C Sr, et al. Masked polycythemia vera: analysis of a single center cohort of 2480 red cell masses. Haematologica. 2019 Aug 14. [Epub ahead of print]

46. Silver RT, Krichevsky S. Distinguishing essential thrombocythemia JAK2V617F from polycythemia vera: limitations of erythrocyte values. Haematologica.2019;104(11):2200-2205.

47. Gisslinger $\mathrm{H}$, Zagriitschuk $\mathrm{O}$, Buxhofer-Ausch V, et al. Ropeginterferon alfa- $2 b$, a novel IFN $\alpha-2 b$, induces high response rates with low toxicity in patients with polycythemia vera. Blood. 2015;126(15):1762-1769.

48. Hasselbalch HC, Holmström MO. Perspectives on interferon-alpha in the treatment of polycythemia vera and related myeloproliferative neoplasms: minimal residual disease and cure? Semin Immunopathol. 2019;41(1):5-19.

49. Silver RT, Gjoni S. The hematocrit value in polycythemia vera: caveat utilitor. Leuk Lymphoma. 2015;56(5):1540-1541.

50. Spivak JL. Polycythemia vera: myths, mechanisms, and management. Blood. 2002;100(13):4272-4290.

51. Pearson TC, Wetherley-Mein G. Vascular occlusive episodes and venous haematocrit in primary proliferative polycythaemia. Lancet. 1978;2(8102):1219-1222

52. Pearson TC. Hemorheologic considerations in the pathogenesis of vascular occlusive events in polycythemia vera. Semin Thromb Hemost. 1997;23(5):433-439.

53. Pearson TC, Guthrie DL, Simpson J, et al. Interpretation of measured red cell mass and plasma volume in adults: Expert Panel on Radionuclides of the International Council for Standardization in
Haematology. Br J Haematol. 1995;89(4):748-756.

54. Marchioli R, Finazzi G, Specchia G, et al. ; CYTO-PV Collaborative Group. Cardiovascular events and intensity of treatment in polycythemia vera. N Engl J Med. 2013;368(1):22-33

55. Warny M, Helby J, Birgens HS, et al. Arterial and venous thrombosis by high platelet count and high hematocrit: 108521 individuals from the Copenhagen General Population Study. J Thromb Haemost. 2019 Jul 15. [Epub ahead of print]

56. Kannel WB, Gordon T, Wolf PA, McNamara P. Hemoglobin and the risk of cerebral infarction: the Framingham study. Stroke. 1972;3(4):409-420.

57. Pedersen KM, Bak M, Sørensen AL, et al. Smoking is associated with increased risk of myeloproliferative neoplasms: A general population-based cohort study. Cancer Med. 2018;7(11):5796-5802.

58. Barosi G, Buratti A, Costa A, et al. An atypical myeloproliferative disorder with high thrombotic risk and slow disease progression. Cancer. 1991;68(10):2310-2318.

59. Osler W. A clinical lecture on erythremia. Lancet. 1908;171(1):143146.

60. Weber FP. Polycythaemia, erythrocytosis and erythraemia. Q J Med. 1908:2:85-134

61. Gordeuk VR, Key NS, Prchal JT. Re-evaluation of hematocrit as a determinant of thrombotic risk in erythrocytosis. Haematologica. 2019 Apr;104(4):653-8.)

62. Nersesjan V, Zervides KA, Sørensen AL, Kjær L, Skov V, Hasselbalch HC. The red blood cell count and the erythrocyte sedimentation rate in the diagnosis of polycythemia vera. Eur J Haematol 2019 Accepted for publication.

\title{
Standing at odds: mutated RAS and hematopoietic stem cells
}

\author{
Monica Nafria and Constanze Bonifer \\ Institute of Cancer and Genomic Sciences, College of Medicine and Dentistry, University of Birmingham, Birmingham, UK \\ E-mail: CONSTANZE BONIFER - c.bonifer@bham.ac.uk
}

doi:10.3324/haematol.2019.230029

A cute myeloid leukemia (AML) is the most common acute leukemia in adults and is characterized by the accumulation of myeloid leukemic blasts unable to complete differentiation. However, AML is a complex disease with variable outcomes and prognoses. ${ }^{1}$ Underlying these heterogeneous phenotypes is the fact that each sub-type of AML is defined by a different set of mutations and is controlled by a specific transcriptional and signaling network distinct to that of normal stem and progenitor cells. ${ }^{2}$ Genes mutated in AML are involved in gene regulation and include transcription factors, chromatin modifiers / remodelers, splicing regulators, DNA methyltransferases and signaling regulators that control the activity of inducible transcription factors. The result is a profound deviation from the normal differentiation trajectory, with each AML sub-type taking a different path and establishing its own cellular identity. ${ }^{2,3}$ Most AML sub-types carry more than one mutation and, with the exception of MLL-translocations (which are a hallmark of pediatric $\mathrm{AML}^{4}$ ), for a number of sub-types it has been shown that the first oncogenic hit is not sufficient to cause overt leukemia. In AML patients, mutations in genes from different functional categories co-exist, and data from sequencing studies as well as mouse models support this notion. ${ }^{5,6}$

The $\mathrm{t}(8 ; 21)$ translocation, occurring in $7 \%$ of adult de novo patients, is one of the most frequent cytogenetic aberrations in AML. 'This translocation fuses the DNAbinding domain coding region of the hematopoietic master regulator RUNX1 (AML1) to the Eight-twenty-One (ETO, RUNX1T1 or MTG8) gene, which encodes a nuclear co-repressor. The result is the formation of the AML1-ETO (alternatively named RUNX1-ETO) chimeric protein, which retains the ability to bind to RUNX1 binding motifs but lacks the transactivation domain of RUNX1., Germline expression of full-length AML1-ETO in mice causes embryonic lethality, ${ }^{10,11}$ but conditional expression in hematopoietic stem cells (HSC) leads to an initial expansion of myeloid progenitor cells, including HSC and granulocyte-macrophage progenitors (GMP). Such expansion was also seen with AML1-ETO-transduced human cord blood-derived HSC in vitro. ${ }^{12}$ Fusion $\mathrm{t}(8 ; 21)$ transcripts have been detected in utero and in postnatal blood samples ${ }^{13}$ and remain expressed at low levels in blood samples from t(8;21) AML patients in long-term remission. ${ }^{14}$ Furthermore, several AML1-ETO-expressing mouse models have failed to fully develop t(8;21) AML unless challenged by mutagenesis or aging, ${ }^{15-18}$ indicating the necessity of additional secondary mutations. These findings suggest that this chromosomal rearrangement is the driver mutation establishing a pre-leukemic clone. This notion is supported by the finding that $t(8 ; 21)$ patients present with a number of different secondary mutations. ${ }^{19}$ The most prominent of these mutations 
occur in genes encoding for the growth factor receptors KIT and FLT3, ${ }^{20,21}$ rendering these receptors chronically active, together with activating RAS mutations such as the K-RAS (G12D), all of which co-operate with AML1ETO. Interestingly, K-RAS activating mutations occur late during leukemia development and are rarely detectable within the leukemic stem cell compartment ${ }^{21}$ (Figure 1A). In myelodysplastic syndromes (MDS), it was shown that the order of acquisition of mutations makes a profound difference on the disease phenotype, ${ }^{22}$ but whether the same stands true for $\mathrm{t}(8 ; 21)$ AML is not known.

In this issue of Haematologica, Di Genua et al. ${ }^{23}$ uncovered the mechanistic explanation for the absence of signaling pathway mutations, such as K-RAS activating mutations, within the $t(8 ; 21)$ pre-leukemic HSC compart- ment by analyzing hematopoietic development and gene expression in conditional murine knock-in models expressing human AML1-ETO and K-RAS(G12D) individually or in combination, resulting in the generation of an $\mathrm{Am}_{1} \mathrm{1}^{\mathrm{ETO} /+}$ (AM), a K-Ras ${ }^{G 12 D /+}(\mathrm{KM})$, and a double-targeted $A m l 1^{E T O /+} ; \mathrm{K}-\mathrm{Ras}^{\mathrm{G} 12 \mathrm{D} /+}$ (AKM) mouse lines. Prior studies had shown that mice expressing $K$-Ras(G12D) from its endogenous locus develop MDS but not AML. ${ }^{24}$ When combined with AML1-ETO in human hematopoietic cells, K-RAS(G12D) promoted leukemic transformation in murine transplantation models. ${ }^{25}$ However, here expression levels were likely to be non-physiological and it had not been defined whether the presence of mutated RAS was compatible with the maintenance of AML1ETO-expressing HSC. Di Genua et al. addressed these

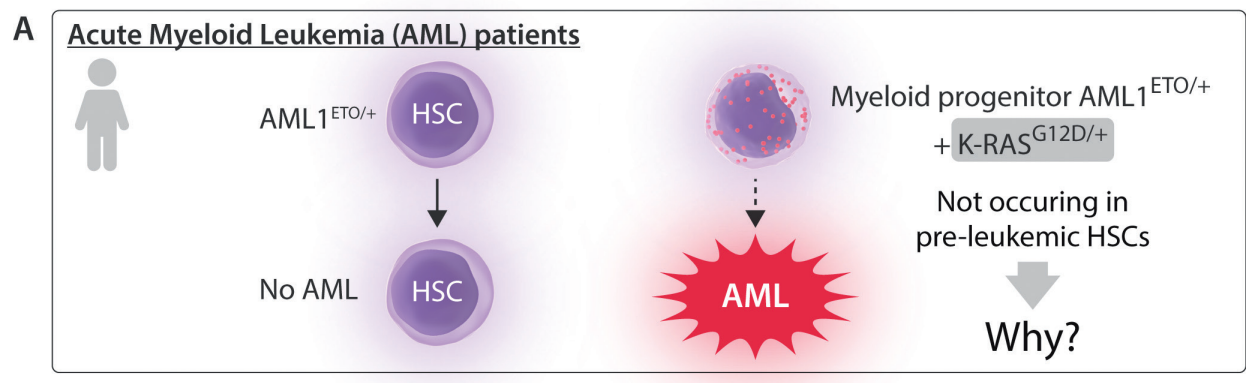

B

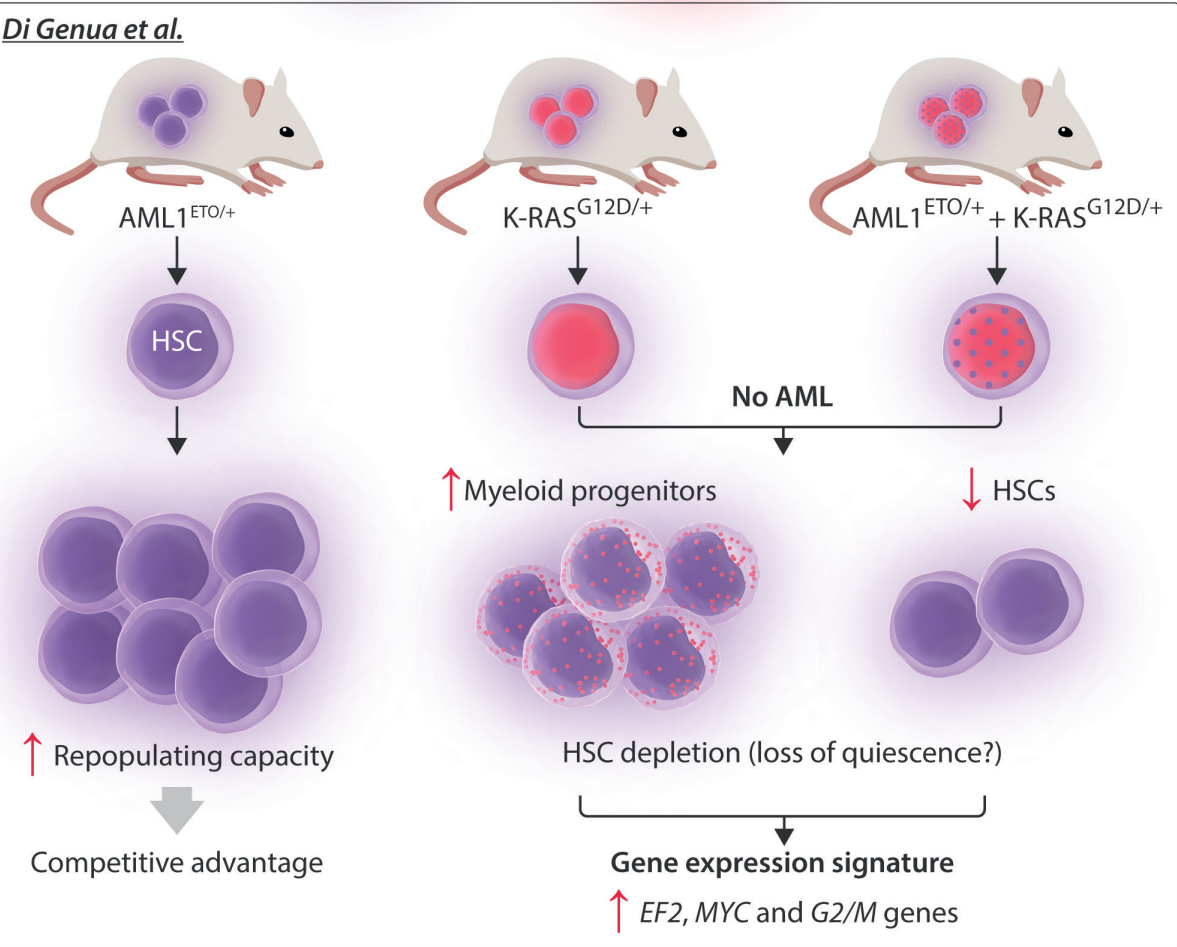

\section{Conclusion}

$1^{\text {st }}$ oncogenic hit

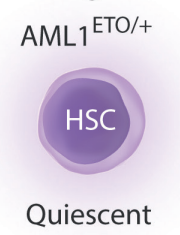

\section{$2^{\text {nd }}$ oncogenic hit} $\mathrm{AML}^{\mathrm{ETO} /+}+\mathrm{K}-\mathrm{RAS}^{\mathrm{G} 12 \mathrm{D} /+}$

Myeloid progenitors

Myeloproliferative phenotype
Additional events?

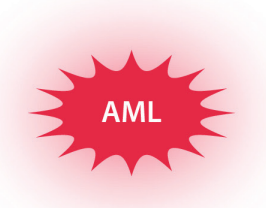

Figure 1. Co-expression of AML1ETO and mutant RAS is incompatible with hematopoietic stem cell (HSC) maintenance. (A) In acute myeloid leukemia (AML) patients, HSC express AML1-ETO, but this is not sufficient to cause overt disease. AML leukemic blasts represent myeloid progenitors harboring the translocation that have undergone secondary oncogenic events, including mutations in signaling pathway genes such as the K$\mathrm{RAS}^{\mathrm{G} 12 \mathrm{D} / *}$. However, prior to Di Genua et $\mathrm{al}_{.}{ }^{23}$ the molecular explanation for the lack of mutations in signaling pathway genes in the pre-leukemic stem cells was unknown. HSC (purple cells), myeloid progenitors (purple cells with pink dots). (B) Di Genua et al. ${ }^{23}$ generated conditional murine knock-in models expressing human AML1-ETO and $K$ $R A S(G 12 D)$ individually or in combination: $A m / 1^{\mathrm{ETO} /+}$ (purple nuclei), K$\operatorname{Ras}^{\mathrm{G120/+}}$ (pink nuclei), and $A m / 1^{\mathrm{ETO} /+}$ K-Ras ${ }^{G 12 D /+}$ (pink and dotted nuclei), respectively, and performed competitive transplantation assays to compare wild-type and transgenic stem cell activity and measure global gene expression as indicated. (C) Model of step-wise oncogenesis in $t(8: 21)$ patients: the presence of $t(8 ; 21)$ in $\mathrm{HSC}$ results in a quiescent phenotype. Acquisition of $\mathrm{K}$ RAS(G12D) occurs at later stages and results in increased proliferation. However, the double oncogenic event is not sufficient to develop overt AML, and the nature and order of acquisition of additional oncogenic events remains unknown. 
issues by conditionally introducing both mutant genes from the respective genomic loci in mice in order to reproduce physiological oncogene expression levels. They then subsequently performed competitive transplantation assays to be able to directly compare stem cell activity in wild-type and transgenic cells (Figure 1B).

AML1-ETO expression on its own affected platelet, Band T-cell development, and led to an increased number of functional HSC together with enhanced myeloid reconstitution in secondary recipients. Expression of mutant K-RAS on its own resulted in a myeloproliferative phenotype, led to a lack of HSC expansion, decreased engraftment and diminished in vitro re-plating potential, which, importantly, was seen regardless of the presence of AML1-ETO. Interestingly, co-expression of both oncoproteins led to a milder myeloproliferative phenotype but not overt AML, as seen in patients, and only aggravated the defect in platelet development. The authors suggest that the competitive advantage observed in AML1-ETOexpressing HSC was due to an enhanced self-renewal capacity. To further elucidate the mechanisms behind the functional impairment of $K$-Ras(G12D)-expressing HSC, they compared gene expression in HSC from genetically modified and control cells using transplantation assays. The presence of mutant K-RAS conferred increased cell cycle activity as well as upregulation of the expression of checkpoint associated genes, such as E2F, Myc and G2Massociated genes, as compared to HSC harboring the AML1-ETO transgene only. Moreover, the presence of mutated K-RAS protein resulted in the downregulation of gene expression signature associated with self-renewal activity and acquisition of a GMP-associated transcriptional signature. Overall, the transcriptional profiles of $\mathrm{K}$ RAS(G12D)-expressing HSC resembled those of myeloid progenitors. Gene expression changes in double mutant cells were distinct from those of cells carrying the individual mutations. For example, two genes, Gja1 and Gzmb, were up-regulated in the double mutant HSC and the authors suggest that they regulate the p53 pathway and oxidative phosphorylation, respectively, both increasing cell death and apoptosis.

Taken together, Di Genua et al. ${ }^{23}$ show that expression of a mutant K-RAS is not compatible with a pre-leukemic state of murine AML1-ETO-expressing HSC. Although AML1-ETO alone confers a competitive advantage to HSC, the additional presence of K-RAS(G12D) results in loss of HSC function, most likely by exhaustion. The study shows that this phenomenon is explained by an increase in cell cycle activity leading to a loss of quiescence in HSC co-expressing both mutations. Therefore, they hypothesize that acquisition of mutant K-RAS in $t(8 ; 21)$ AML occurs at the myeloid progenitor stage rather than within pre-leukemic HSC compartment harboring the $t(8 ; 21)$ translocation. Overall, this study demonstrates that the order of appearance of each class of mutations is also relevant for leukemic transformation in AML.

Additional questions arise from this and previous work (Figure 1C). Firstly, Di Genua et al. show that, even in the presence of K-RAS(G12D), AML1-ETO is unable to cause an overt leukemic phenotype in vivo. The same was found in xenotransplantation experiments with retrovirally transduced human $\mathrm{CD} 34^{+}$cord blood cells expressing
AML1-ETO together with N-RAS(G12D). ${ }^{26}$ This notion ties in with the observation of Cabezas-Wallscheid et al. ${ }^{16}$ that the development of AML in an AML1-ETO mouse model is a slow process, probably requiring multiple mutations or epigenetic reprogramming events, which were not examined in their study. Moreover, it has been previously shown that expression of AML1-ETO in an inducible transgenic mouse model leads to a block in differentiation, but not to an enhanced proliferation. ${ }^{17} \mathrm{Di}$ Genua et al. ${ }^{23}$ add to this result that AML1-ETO-expressing pre-leukemic stem cells have a quiescent phenotype, leading to an accumulation of cells with high re-plating activity that are capable of going into cycle in a transplantation setting. Given that the double oncogenic event studied here is not enough to cause AML, the question that is now apparent is which additional events cause AML in patients. This work demonstrates that, even with a type of AML that has been studied in fine molecular detail for decades, the players of selection and clonal evolution in patients still have additional cards up their sleeve.

\section{References}

1. De Kouchkovsky I, Abdul-Hay M. Acute myeloid leukemia: a comprehensive review and 2016 update. Blood Cancer J. 2016;6(7):e441.

2. Assi SA, Imperato MR, Coleman DJL, et al. Subtype-specific regulatory network rewiring in acute myeloid leukemia. Nat Genet. 2019;51(1):151-162.

3. Valk PJ, Verhaak RG, Beijen MA, et al. Prognostically useful geneexpression profiles in acute myeloid leukemia. $N$ Engl J Med. 2004;350(16):1617-1628.

4. Szczepanski T, Harrison CJ, van Dongen JJ. Genetic aberrations in paediatric acute leukaemias and implications for management of patients. Lancet Oncol. 2010;11(9):880-889.

5. Papaemmanuil E, Gerstung M, Bullinger L, et al. Genomic Classification and Prognosis in Acute Myeloid Leukemia. N Engl J Med. 2016;374(23):2209-2221.

6. Wang M, Yang C, Zhang L, Schaar DG. Molecular Mutations and Their Cooccurrences in Cytogenetically Normal Acute Myeloid Leukemia. Stem Cells Int. 2017;2017:6962379.

7. Grimwade D, Hills RK, Moorman AV, et al. Refinement of cytogenetic classification in acute myeloid leukemia: determination of prognostic significance of rare recurring chromosomal abnormalities among 5876 younger adult patients treated in the United Kingdom Medical Research Council trials. Blood. 2010;116(3):354-365.

8. Miyoshi H, Shimizu K, Kozu T, Maseki N, Kaneko Y, Ohki M. $\mathrm{t}(8 ; 21)$ breakpoints on chromosome 21 in acute myeloid leukemia are clustered within a limited region of a single gene, AML1. Proc Natl Acad Sci U S A. 1991;88(23):10431-10434.

9. Erickson P, Gao J, Chang KS, et al. Identification of breakpoints in $t(8 ; 21)$ acute myelogenous leukemia and isolation of a fusion transcript, AML1/ETO, with similarity to Drosophila segmentation gene, runt. Blood. 1992;80(7):1825-1831.

10. Yergeau DA, Hetherington CJ, Wangg Q, et al. Embryonic lethality and impairment of haematopoiesis in mice heterozygous for an AML1-ETO fusion gene. Nat Genet. 1997;15(3):303-306.

11. Cai Z, Yang S, Okuda T, et al. Expression of a knocked-in AML1ETO leukemia gene inhibits the establishment of normal definitive hematopoiesis and directly generates dysplastic hematopoietic progenitors. Blood. 1998;91(9):3134-3143.

12. Mulloy JC, Cammenga J, MacKenzie KL, Berguido FJ, Moore MA, Nimer SD. The AML1-ETO fusion protein promotes the expansion of human hematopoietic stem cells. Blood. 2002;99(1):15-23.

13. Wiemels JL, Xiao Z, Buffler PA, et al. In utero origin of $t(8 ; 21)$ AML1ETO translocations in childhood acute myeloid leukemia. Blood. 2002;99(10):3801-3805.

14. Miyamoto T, Weissman IL, Akashi K. AML1/ETO-expressing nonleukemic stem cells in acute myelogenous leukemia with 8;21 chromosomal translocation. Proc Natl Acad Sci U S A. 2000;97(13):75217526.

15. Higuchi M, O'Brien D, Kumaravelu P, Lenny N, Yeoh EJ, Downing JR. Expression of a conditional AML1-ETO oncogene bypasses 
embryonic lethality and establishes a murine model of human $\mathrm{t}(8 ; 21)$ acute myeloid leukemia. Cancer Cell. 2002;1(1):63-74.

16. Cabezas-Wallscheid N, Eichwald V, de Graaf J, et al. Instruction of haematopoietic lineage choices, evolution of transcriptional landscapes and cancer stem cell hierarchies derived from an AML1-ETO mouse model. EMBO Mol Med. 2013;5(12):1804-1820.

17. Rhoades KL, Hetherington CJ, Harakawa N, et al. Analysis of the role of AML1-ETO in leukemogenesis, using an inducible transgenic mouse model. Blood. 2000;96(6):2108-2115.

18. Yuan Y, Zhou L, Miyamoto T, et al. AML1-ETO expression is directly involved in the development of acute myeloid leukemia in the presence of additional mutations. Proc Natl Acad Sci U S A. 2001;98(18):10398-10403.

19. Christen F, Hoyer K, Yoshida K, et al. Genomic landscape and clonal evolution of acute myeloid leukemia with $\mathrm{t}(8 ; 21)$ : an international study on 331 patients. Blood. 2019;133(10):1140-1151.

20. Kuchenbauer F, Schnittger S, Look T, et al. Identification of additional cytogenetic and molecular genetic abnormalities in acute myeloid leukaemia with t(8;21)/AML1-ETO. Br J Haematol. 2006;134(6):616-619.

21. Corces-Zimmerman MR, Majeti R. Pre-leukemic evolution of hematopoietic stem cells: the importance of early mutations in leukemogenesis. Leukemia. 2014;28(12):2276-2282.

22. Shima T, Miyamoto T, Kikushige Y, et al. The ordered acquisition of Class II and Class I mutations directs formation of human $t(8 ; 21)$ acute myelogenous leukemia stem cell. Exp Hematol. 2014;42(11) :955-965.e1-5.

23. Di Genua C, Norfo R, Rodriguez-Meira A, et al. Cell-intrinsic depletion of Aml1-ETO-expressing pre-leukemic hematopoietic stem cells by K-Ras activating mutation. Haematologica. 2019;104(11):22152224.

24. Braun BS, Tuveson DA, Kong N, et al. Somatic activation of oncogenic Kras in hematopoietic cells initiates a rapidly fatal myeloproliferative disorder. Proc Natl Acad Sci U S A. 2004;101(2):597-602.

25. Zhao S, Zhang Y, Sha K, et al. KRAS (G12D) cooperates with AML1/ETO to initiate a mouse model mimicking human acute myeloid leukemia. Cell Physiol Biochem. 2014;33(1):78-87.

26. Chou FS, Wunderlich M, Griesinger A, Mulloy JC. N-Ras(G12D) induces features of stepwise transformation in preleukemic human umbilical cord blood cultures expressing the AML1-ETO fusion gene. Blood. 2011;117(7):2237-2240.

\title{
Carfilzomib combination treatment as first-line therapy in multiple myeloma: where do we go from the Carthadex (KTd)-trial update?
}

\author{
Monika Engelhardt, ${ }^{1,2}$ Kwee Yong, ${ }^{3}$ Sara Bringhen ${ }^{4}$ and Ralph Wäsch ${ }^{1,2}$ \\ ${ }^{1}$ Hematology and Oncology, Faculty of Medicine, University of Freiburg, Freiburg, Germany; ${ }^{2}$ Comprehensive Cancer Center Freiburg \\ (CCCF), Freiburg, Germany; ${ }^{3}$ University College London, London, UK and ${ }^{4}$ UNITO Dipartimento di Oncologia, University of Turin, \\ Turin, Italy
}

E-mail: MONIKA ENGELHARDT - monika.engelhardt@uniklinik-freiburg.de

doi:10.3324/haematol.2019.228684

T

he prognosis and treatment of multiple myeloma (MM) patients have substantially changed in the last decade due to a better understanding of the disease and the introduction of novel agents (NA) with new mechanisms of action against malignant plasma cells. ${ }^{1-3}$ In parallel with the improved understanding of myeloma biology, the field has witnessed a flood of NA, including immunomodulatory drugs (IMiD: thalidomide, lenalidomide, pomalidomide); proteasome inhibitors (PI: bortezomib, carfilzomib, ixazomib), monoclonal antibodies (mAb: daratumumab, elotuzumab), ${ }^{4}$ and histone deacetylase inhibitors, which have substantially improved progression-free survival (PFS) and overall survival (OS). Other NA in clinical trials (selinexor, venetoclax, novel immunotherapeutics, iberdomide, and others) are being intensively tested, and specifically immunotherapeutics beyond $\mathrm{mAb}$, such as bispecific $\mathrm{T}$ cell engager (BITE) molecules and chimeric antigen receptor (CAR)-T cells will expand anti-myeloma therapy options. ${ }^{1-4}$ Concomitantly, the application of tools that reliably assess "frailty" of patients is also helping with decision making, given that many patients with $\mathrm{MM}$ are elderly and often have significant comorbidities. ${ }^{5-10}$ Sustained disease response is crucial in fit and in frail patients, since disease response can significantly improve quality of life and may reduce MM-induced comorbidity. Optimizing tolerability for timely treatment delivery has also proved beneficial. ${ }^{11}$ However, this may prove challenging with triplet or quadruplet regimens that are being developed for continued therapy, where adverse events (AE) may lead to treatment interruptions and discontinuation.
After the introduction of the first PI bortezomib (Btz/V), second- and third-generation PI were developed, with the aim of providing therapy that would be potentially more efficacious and less toxic, including an improved polyneuropathy (PNP) side effect profile. Carfilzomib $(\mathrm{Cfz} / \mathrm{K})$ is a second-in-class, epoxyketonebased, irreversibly binding PI, which is approved in combination with dexamethasone $(\mathrm{Kd})$ or lenalidomide and dexamethasone (KRd) for the treatment of relapsed/refractory MM (RRMM) patients. ${ }^{12,13}$ The ENDEAVOR study compared Kd versus Btz plus dexamethasone $(\mathrm{Vd})$ and reported a longer PFS and OS, with lower risk of painful PNP with $\mathrm{Kd} .{ }^{13}$ The ASPIRE study demonstrated the superiority of $\mathrm{KRd}$ over $\mathrm{Rd}$, with unprecedented PFS benefit, as well as OS benefit in RRMM. ${ }^{14}$ These studies have established the place of Cfz in treating RRMM.

Dyspnea, hypertension and cardiac toxicities stand out as clinically relevant side effects, and a widening experience of these has led to published guidance for the use of $\mathrm{Cfz}$, as well as a re-appraisal of the baseline cardiovascular morbidity present in this patient group. ${ }^{15}$ Such guidance provides a helpful description of expected events, as well as suggestions for subsequent monitoring, detection and management. ${ }^{16,17}$ The analysis of cardiovascular adverse events (CVAE) in Cfz-treated patients revealed that, in those with CVAE, 91\% had uncontrolled hypertension, with acute coronary syndrome or cardiac arrhythmias each present in $4.5 \%$. Subjects with CVAE also had significantly higher blood pressure, left ventricular mass, and pulse wave velocity at baseline evaluation, compared to those without. Baseline uncontrolled blood 